\title{
Reinforcement of graphene nanoplatelets on plasticized poly (lactic acid) nanocomposites: mechanical, thermal, morphology, and antibacterial properties
}

\begin{abstract}
Plasticized poly(lactic acid) (PLA)-based nanocomposites filled with graphene nanoplatelets $(\mathrm{xGnP})$ and containing poly(ethylene glycol) (PEG) and epoxidized palm oil (EPO) with ratio $2: 1(2 \mathrm{P}: 1 \mathrm{E})$ as hybrid plasticizer were prepared by melt blending method. The key objective is to take advantage of plasticization to increase the material ductility while preserving valuable stiffness, strength, and toughness via addition of $\mathrm{xGnP}$. The tensile modulus of PLA/2P : 1E/0.1 wt \% xGnP was substantially improved (30\%) with strength and elasticity maintained, as compared to plasticized PLA. TGA analysis revealed that the $\mathrm{xGnP}$ was capable of acting as barrier to reduce thermal diffusion across the plasticized PLA matrix, and thus enhanced thermal stability of the plasticized PLA. Incorporation of $\mathrm{xGnP}$ also enhanced antimicrobial activity of nanocomposites toward Escherichia coli, Salmonella typhimurium, Staphylococcus aureus, and Listeria monocytogenes.
\end{abstract}

Keywords: Mechanical properties; Morphology; Polyesters; Properties and characterization; Thermogravimetric analysis (TGA) 